\title{
Suicide and Accidental Death Among Women with Primary Ovarian Cancer: A population-based study
}

\author{
Ying Chen ${ }^{1}$, Kaixu Yu ${ }^{1}$, Qingqing Zhu ${ }^{1}$, Weicheng Tang ${ }^{1}$, Dan Chen ${ }^{1}$, Liru Xue ${ }^{1}$, Jinjin \\ Zhang $^{1}$, Su Zhou ${ }^{1}$, Jun Dai ${ }^{1}$, Yan Jin ${ }^{1}$, Meng $\mathrm{Wu}^{1}$, and SHIXUAN WANG ${ }^{1}$ \\ ${ }^{1}$ Affiliation not available
}

May 8, 2021

\begin{abstract}
Objective To determine the relative risk of suicide and accidental death among patients with ovarian cancer to that of the general population, and to identify risk factors associated with suicide and accidental death. Design The surveillance, epidemiology, and end results (SEER) registry provided ovarian cancer data from 18 registries. Setting Surveillance, Epidemiology, and End Results database. Population The study population comprised 149,204 patients. Methods The standardized mortality ratios (SMRs) were calculated and Fine-Gray models were fitted, with stratifications on demographic and tumor-related characteristics. Main outcome measures The standardized mortality ratios. Results Women with ovarian cancer had a higher risk of suicide and accidental death than the cancer-free group (SMR=1.86; 95\% CI [1.54-2.25] and SMR=1.54; 95\% CI [1.39-1.71]). Subgroup analysis indicated that only patients with type II epithelial ovarian cancer (SMR=2.31; 95\% CI [1.83-2.91]) had an increased risk of suicide, and those with type I and type II epithelial ovarian cancer (SMR=1.65; 95\% CI [1.39-1.97] and SMR=1.49; 95\% CI [1.30-1.70]) were at a higher risk of accidental death. Patients with ovarian cancer who were younger, white, diagnosed with high-grade, non-metastatic cancer and pelvic exenteration were at a higher risk of suicide. Additionally, pelvic exenteration increased the risk of suicide but not the risk of accidental death among these women. Conclusion Women with ovarian cancer had a higher risk of suicide and accidental death compared with the general population. Clinicians should identify high-risk subgroups of ovarian cancer patients for suicide and accidental death as early as possible, with appropriate prevention strategies.
\end{abstract}

Title: Suicide and Accidental Death Among Women with Primary Ovarian Cancer: A population-based study

\section{Running title: Suicide and Ovarian Cancer}

\section{Authors:}

Ying Chen ${ }^{\text {a, }}$, Kaixu Yu ${ }^{\text {b, }}{ }^{1}$, Qingqing Zhu ${ }^{\text {a }}$, Weicheng Tang ${ }^{a}$, Dan Chen ${ }^{\text {a }}$, Liru Xue ${ }^{a}$, Jinjin Zhang ${ }^{\text {a }}$, Su Zhou ${ }^{\mathrm{a}}$, Jun Dai ${ }^{\text {a }}$, Yan Jin ${ }^{\mathrm{a}}$, Meng Wu ${ }^{\text {a, }}{ }^{*}$, Shixuan Wanga, *

\section{Affiliations:}

${ }^{a}$ Department of Obstetrics and Gynaecology, Tongji Hospital, Tongji Medical College, Huazhong University of Science and Technology, Wuhan 430022, China

b Department of Orthopedics, Tongji Hospital, Tongji Medical College, Huazhong University of Science and Technology, Wuhan 430022, China

1 These authors contributed equally.

\section{* Correspondence:}


Shixuan Wang, PhD, Department of Obstetrics and Gynaecology, Tongji Hospital, Tongji Medical College, Huazhong University of Science and Technology, Wuhan 430022, China. Email:sxwang@tjh.tjmu.edu.cn. Telephone number: +86-139-9555-3319. Fax number: 86-27-83663087

Meng Wu, PhD, Department of Obstetrics and Gynaecology, Tongji Hospital, Tongji Medical College, Huazhong University of Science and Technology, Wuhan 430022, China.

Email:mengwu@tjh.tjmu.edu.cn. Telephone number: +86-159-2712-2310. Fax number: 86-27-83662407

Word count: 3794

\section{ABSTRACT:}

\section{Objective}

To determine the relative risk of suicide and accidental death among patients with ovarian cancer to that of the general population, and to identify risk factors associated with suicide and accidental death.

\section{Design}

The surveillance, epidemiology, and end results (SEER) registry provided ovarian cancer data from 18 registries.

\section{Setting}

Surveillance, Epidemiology, and End Results database.

\section{Population}

The study population comprised 149,204 patients.

\section{Methods}

The standardized mortality ratios (SMRs) were calculated and Fine-Gray models were fitted, with stratifications on demographic and tumour-related characteristics.

\section{Main outcome measures}

The standardized mortality ratios.

\section{Results}

Women with ovarian cancer had a higher risk of suicide and accidental death than the cancer-free group $(\mathrm{SMR}=1.86 ; 95 \%$ CI $[1.54-2.25]$ and $\mathrm{SMR}=1.54 ; 95 \%$ CI [1.39-1.71]). Subgroup analysis indicated that only patients with type II epithelial ovarian cancer $(\mathrm{SMR}=2.31 ; 95 \%$ CI [1.83-2.91]) had an increased risk of suicide, and those with type I and type II epithelial ovarian cancer (SMR=1.65; 95\% CI [1.39-1.97] and $\mathrm{SMR}=1.49 ; 95 \%$ CI [1.30-1.70]) were at a higher risk of accidental death. Patients with ovarian cancer who were younger, white, diagnosed with high-grade, non-metastatic cancer and pelvic exenteration were at a higher risk of suicide. Additionally, pelvic exenteration increased the risk of suicide but not the risk of accidental death among these women.

\section{Conclusion}

Women with ovarian cancer had a higher risk of suicide and accidental death compared with the general population. Clinicians should identify high-risk subgroups of ovarian cancer patients for suicide and accidental death as early as possible, with appropriate prevention strategies.

\section{Word count: 249}

\section{Keywords}

Ovarian cancer, Suicide and accidental death, Histological subtypes, Pelvic exenteration, Risk factor 


\section{Tweetable abstract}

Women with ovarian cancer had a higher risk of suicide and accidental death compared with the general population.

\section{Introduction}

Suicide is the tenth leading cause of death in the US, claiming the lives of more than 48,000 individuals in $2018^{1}$. It not only represents an individual tragedy but also takes a staggering toll on global public health. The hitherto unknown suicidal deaths may have been classified as death from accidental or unintentional injury $^{23}$. Both suicide and accidental death are regarded as external causes of death, and they share common risk factors such as physical, social, and emotional dysfunction ${ }^{4}$. Though the 5 -year survival rates of cancer patients have increased over the past decades, the risk of dying from suicide and accidental death for those patients remains elevated over time ${ }^{5}$. Epidemiological studies have shown that the rate of death from suicide and unintentional injury in cancer patients was $1.9{ }^{5}$ and $1.6{ }^{6}$ times those of the general population, respectively.

Gynaecologic cancers represent a major problem affecting women's health. Kristy et al. reported that women with gynaecological malignancies were at a higher risk of suicide compared with those diagnosed with nongynaecological malignancies ${ }^{7}$. Among gynaecologic cancers, the patients with ovarian cancer had the highest suicidal rate among patients with gynaecological malignancies ${ }^{8}$. It was estimated that approximately $75 \%$ of ovarian cancer occurs as an advanced disease ${ }^{9}$ because of the lack of effective screening methods and non-specific symptoms ${ }^{10}$. This resulted in a high recurrence and mortality rate in ovarian cancer patients. Severe pain, high recurrence rate, intense treatment, and inferior quality of life from the disease itself and adverse effects of treatment often caused serious depression and anxiety among patients with ovarian cancer, which could put them at high risk for suicide and accidental death ${ }^{11}$.

Previous studies have shown higher suicide rates in ovarian cancer patients compared with other gynaecological malignancies ${ }^{8}$, however, a direct comparison of the risk of suicide and accident injury especially among ovarian cancer with the general population has not been undertaken, and there is currently no literature on characteristics, incidence, and risk factors for suicide and accidental death in women with ovarian cancers in details.

As such, to address the current lack of evidence, we conducted a population-based analysis of the suicide and accidental death among patients with ovarian cancer. The objective of this study was to determine the relative risk of suicide and accidental deaths among ovarian cancer patients compared with the general population and to identify demographic and tumour-related characteristics, such as age at diagnosis and histological subtypes, that is associated with a particularly high risk of suicide and accidental deaths among ovarian cancer patients.

\section{Methods}

The Surveillance, Epidemiology, and End Results (SEER) database ${ }^{12}$ was established by the National Cancer Institute, covering approximately $28 \%$ of the US population. The Public Use version of data collected from the SEER18 registries ${ }^{12}$ between January 1, 1973, and December 31, 2016, was used for this study. As a comparison, the mortality data of the general US population collected by the National Centre for Health Statistics spanning from 1969 to 2016 was used.

To avoid the influence of the second primary tumours on the outcome of cancer patients, we only included patients diagnosed with the first primary ovarian cancer at the beginning of this study. Patients were excluded if the diagnosis was made at autopsy or obtained solely from the death certificate, and those without definite data on age at diagnosis, income, educational level, and survival time were also excluded in this study (Supplementary Figure.1).

Available data about demographic characteristics from the SEER database included age at diagnosis, race, and calendar year of diagnosis. The tumour-related variables encompassed pathological type, stage, and 
grade. Income (median family income) and educational level (percentage of residents $>25$ years of age with at least a bachelor's degree) were categorized into quartiles. Residence types (rural/urban) were obtained at the county level from the SEER program. Data on ovarian cancer treatment, follow-up time, and cause of death were also available. Ovarian cancer was categorized into five histological subgroups according to the International Classification of Disease for Oncology third revision (ICD-O-3) codes: type I epithelial, type II epithelial, germ cell, sex cord-stromal, and other ${ }^{1314}$. Surgeries for ovarian cancer were categorized into "Oophorectomy without hysterectomy", "Oophorectomy with hysterectomy", "Surgery, not otherwise specified (NOS)", "None", "Debulking", and "Pelvic Exenteration".

Patients with the following causes of death codes in the SEER database were considered to have committed suicide: International Classification of Diseases, Eighth Revision codes (ICD-8): 950-959; International Classification of Diseases, Ninth Revision codes (ICD-9): 950-959; and International Statistical Classification of Diseases and Related Health Problems, Tenth Revision (ICD-10) codes U03, X60-X84, and Y87.0 and recode 50220. Patients with the following causes of death codes in the SEER database were considered to have committed accidental death: ICD-8: 800-949; ICD-9: 800-949; and ICD-10: V01-X59 and Y85-Y86 and recode 50210 .

The number of suicides or accidental deaths divided by person-years of survival was calculated as the mortality of suicidal or accidental death. Among subgroups of cancer patients stratified by different characteristics, SMRs were calculated as the ratios of observed to expected number of deaths ${ }^{5}{ }^{15}{ }^{6}$, which provided the relative risk of death from suicide and accident injury for cancer patients compared with all general US population with the same distribution of age, sex, and race. The observed values represented the number of suicides or accidental deaths in patients with ovarian cancer, and the expected values represented the number of individuals who died by suicide or accident in the general population. A five-year age range was used for standardization, and the 95\% CI of SMR was determined by using the Poisson distribution approximation. Interaction tests were further carried out to investigate the potential differential effect of cancer subtypes on suicide risk during different follow-up periods. Given that other causes of death were considered as competing events, we also designed the Fine-Gray model to identify demographic and tumour-related characteristics associated with a higher risk of suicide and accidental death. Observations were censored if patients did not die from suicide or accidental death at the time of the last follow-up. The survival time recorded as 0 month in the SEER database was converted to one-half of a month based on accepted epidemiologic practices ${ }^{15}$.

All statistical tests were two-sided, and values with $\mathrm{P}<0.05$ were considered statistically significant. The SEER database was accessed using SEER*Stat software 8.3.8 ${ }^{12}$. The calculation of SMRs, cumulative incidence curves, Gray tests, and interaction tests were conducted in $\mathrm{R}$ version 3.51 statistical software. These histograms summarizing the results of subgroup analyses were drawn by Microsoft Excel and GraphPad Prism 8.0.

\section{Results}

\section{Incidence of Suicide and Accidental Death Among Patients with Ovarian Cancer}

A total of 107 suicidal and 373 accidental deaths occurred among 149,204 patients with ovarian cancer, followed by 768,840.17 person-years (Table.1 ). The suicide rate was 13.92/100,000 person-years and the accidental death rate was $48.51 / 100,000$ person-years among ovarian cancer patients. Women with ovarian cancer had an $86 \%$ higher risk of suicidal death (SMR=1.86; 95\% CI [1.54-2.25]) and $54 \%$ higher risk of accidental death $(\mathrm{SMR}=1.54 ; 95 \%$ CI [1.39-1.71]) than the general US population with the same distribution of age, sex, and race. The number of suicide and accidental deaths among patients with ovarian cancer ranks second in patients with gynaecologic cancers, between uterine cancer and cervical cancer. From 1973 to 2017, the relative number of suicide and accidental deaths decreased gradually in patients with uterine cancer and unchanged among patients with cervical cancer, while the relative number of suicide and accidental deaths remained elevated among patients with ovarian cancer (Supplementary Figure.2 ).

Demographic Characteristics Associated with Increased Incidence of Suicide and Accidental Death 
There were 27 suicides and 77 accidental deaths among patients with ovarian cancer who were younger than 50 years old. These younger cancer patients had almost the same risk of suicide as the general population with the same distribution of age, sex, and race ( $\mathrm{SMR}=1.25 ; 95 \%$ CI [0.86-1.83]). But a significantly higher incidence of suicide and accidental death in patients over 50 were observed. Among cancer patients diagnosed between 50 and 59 years old, there were 36 suicides of 32,298 patients, accounting for $33.6 \%$ of all suicides among patients with ovarian cancer (Supplementary Table.1). While for patients whose age at diagnosis was more than 50 years old, the suicide risk gradually decreased with age but increased after the age of 80.Supplementary Figure.3 showed the number and proportion of suicides and accidental deaths in patients with ovarian cancer of different histological subtypes at different ages.

Among all patients with ovarian cancer, white patients had the highest risk of suicide ( $\mathrm{SMR}=1.82 ; 95 \%$ CI [1.50-2.23]) and black patients had the highest risk of accidental death (SMR=1.87; 95\% CI [1.27-2.75]). The predominant patients who committed suicide were those with high education level $(42,39 \%)$, high income (43, 40\%), and metropolitan residents (97, 91\%), with an SMR of 2.12 (95\% CI [1.57-2.87]), 2.05 (95\% CI [1.52-2.77]), and 1.93 (95\% CI [1.58-2.35]), respectively.

Tumour-related Characteristics Associated with Higher Risk of Suicide and Accidental Death

Patients with high-grade ovarian cancer had a higher risk of suicide than those with low-grade ovarian cancer $(17.88$ /100,000 person-years; SMR=2.37; $95 \%$ CI [1.77-3.17] vs 7.23/100,000 person-years; $\mathrm{SMR}=0.94 ; 95 \%$ CI [0.59-1.49]), while there was no difference in the risk of accidental death among the two groups (44.10 $/ 100,000$ person-years; SMR $=1.32 ; 95 \%$ CI [1.10-1.59] and 41.78 /100,000 person-years; SMR=1.48; $95 \%$ CI [1.22-1.80]). Patients with regional cancer and those with distant cancer were more likely to die from suicide (15.19 /100,000 person-years; SMR=2.02; 95\% CI [1.24-3.30] and 16.02 /100,000 person-years; SMR=2.15; $95 \%$ CI [1.63-2.85]), while patients with localized ovarian cancer $(9.82 / 100,000$ person-years; SMR=1.31; 95\% CI [0.92-1.85]) had the same risk of suicide as their matched general population. Moreover, patients with distant ovarian cancer had the highest risk of accidental death (54.91 /100,000 person-years; SMR $=1.59 ; 95 \%$ CI [1.37-1.85]), and patients with localized ovarian cancer and those with regional ovarian cancer had the same risk of accidental death $(41.11 / 100,000$ person-years; SMR=1.50; 95\% CI [1.27-1.78]; $44.61 / 100,000$ person-years; 95\% CI [1.05-1.86]).

\section{Tumour Treatment Associated with Higher Suicidal and Accidental Death Risk}

The suicide rate of patients who did not undergo surgery for ovarian cancer (SMR=6.75; 95\% CI [4.07-11.20]) was much greater than that among those who underwent surgery (SMR=1.57; 95\% CI [1.27-1.94]). Among women who received surgical treatment, those who underwent pelvic exenteration had the least number of cases but the highest risk of suicide $(29.67 / 100,000$ person-years; SMR=3.88; $95 \%$ CI [1.25-12.03]). Patients who underwent oophorectomy with hysterectomy had a higher risk of suicide as compared to those who underwent oophorectomy without hysterectomy (11.24 /100,000 person-years; SMR=1.42; 95\% CI [1.01$2.00]$ vs $6.82 / 100,000$ person-years; $\mathrm{SMR}=1.09 ; 95 \%$ CI [0.52-2.28]). In addition, patients who did not undergo surgery for ovarian cancer and those who underwent oophorectomy with hysterectomy had a higher risk of accidental death compared with their matched general population $(149.88 / 100,000$ person-years; $\mathrm{SMR}=2.32 ; 95 \%$ CI [1.77-3.04] and 40.20 /100,000 person-years; SMR=1.37; 95\% CI [1.14-1.64]).

\section{Suicidal and Accidental Death Risk in Different Histological Subtypes of Ovarian Cancer}

Only patients with Type II epithelial ovarian cancer had an increased risk of suicide, and those with Type I and Type II epithelial ovarian cancer were at a higher risk of accidental death compared with the general population (Figure.1) . Among 102,418 patients with type II ovarian cancer, there were 72 suicides and 214 accidental deaths, accounting for $67 \%$ and $57 \%$ of all suicides and accidental deaths among ovarian cancer patients, respectively (Table.2 ). These patients had the highest risk of suicide and accidental deaths among all patients with ovarian cancer (17.49/100,000 person-years; SMR=2.31; 95\% CI [1.83-2.91] and $51.98 / 100,000$ person-years; SMR=1.49; $95 \%$ CI [1.30-1.70]), followed by those with sex cord ovarian cancer for suicide $(16.55 / 100,000$ person-years; SMR $=2.37 ; 95 \%$ CI [0.89-6.32]) and those with type I ovarian cancer for accidental death $(46.68 / 100,000$ person-years; SMR=1.65; 95\% CI [1.39-1.97]). Patients 
with ovarian cancer of sex cord, type I, and germ cell had an almost equal risk of suicide $(16.55 / 100,000$ person-years; SMR=2.37; 95\% CI [0.89-6.32], 8.82 /100,000 person-years; SMR=1.12; 95\% CI [0.75-1.66], and $7.84 / 100,000$ person-years; SMR $=1.67 ; 95 \% \mathrm{CI}$ [0.63-4.44]) and patients with ovarian cancer of sex cord and germ cell had an almost equal risk of accidental death compared with the general population (41.39 $/ 100,000$ person-years; $\mathrm{SMR}=1.53 ; 95 \%$ CI [0.82-2.85] and 25.48/100,000 person-years; SMR=1.40; $95 \%$ CI [0.81-2.40]). For type II ovarian cancer patients, those with undifferentiated ovarian cancer had the highest risk of suicide and accidental death $(23.94 / 100,000$ person-years; SMR $=3.26 ; 95 \%$ CI $[2.18-4.86]$ and 77.80 /100,000 person-years; SMR=1.94; 95\% CI [1.56-2.43]), followed by those with high-grade serous (15.24 /100,000 person-years; SMR=1.99; 95\% CI [1.48-2.69] and 43.23 /100,000 person-years; SMR=1.30; $95 \%$ CI [1.09-1.56]) and mixed epithelial-stromal carcinoma (17.11/100,000 person-years; SMR $=2.25 ; 95 \%$ CI [0.94-5.41] and 47.90 /100,000 person-years; SMR=1.39; 95\% CI [0.82-2.35]).

\section{Internal Comparisons: Predictors of Suicide and Accidental Death Based on Fine-Gray Model}

The Fine-Gray model revealed that age was found to be a protective factor for suicide $(\mathrm{HR}=0.98 ; 95 \%$ CI [0.97-0.99]; $\mathrm{P}<0.001)$ but an independent risk factor for accidental death (HR=1.02; 95\% CI [1.01-1.03]; $\mathrm{P}<0.001$ ) among patients with ovarian cancer (Supplementary Table.2 ). Patients with high-grade ovarian cancer had a higher risk of suicide rather than accidental death than those with low-grade ovarian cancer. Besides, patients with localized ( $\mathrm{HR}=1.85 ; 95 \%$ CI [1.11-3.08]; $\mathrm{P}=0.019$ and $\mathrm{HR}=2.08 ; 95 \%$ CI [1.56-2.78]; $\mathrm{P}<0.001)$ or regional ovarian cancer $(\mathrm{HR}=1.97 ; 95 \% \mathrm{CI}[1.12-3.47]$; $\mathrm{P}=0.019$ and $\mathrm{HR}=1.65 ; 95 \%$ CI $[1.19$ 2.19]; $\mathrm{P}=0.003$ ) had relatively higher risk of suicide and accidental death compared with those with distant ovarian cancer. The risk of suicide among patients who underwent pelvic exenteration $(\mathrm{HR}=4.14 ; 95 \%$ CI [1.07-15.98]; $\mathrm{P}=0.039)$ for ovarian cancer was approximately five times that of patients who underwent oophorectomy without hysterectomy, while there was no statistical difference in the risk of accidental death in the two groups $(\mathrm{HR}=1.03 ; 95 \% \mathrm{CI}[0.40-2.62] ; \mathrm{P}=0.960)$.Figure.2 showed the cumulative incidence of suicide and accidental death among patients who underwent different types of surgery throughout followup. Moreover, patients with localized or regional ovarian cancer had a significantly high risk of suicide and accidental death. The high-grade cancer population had a higher rate of suicide risk while the risk of accidental death was the same as that of the low-grade cancer population.

\section{Suicidal and Accidental Death Risk Over Time After Diagnosis}

The relative increase in suicide risk among ovarian cancer patients was the highest within the first year after initial diagnosis and subsided with longer follow-up time (Supplementary Table.3 ). Ten years after the cancer diagnosis, the suicide risk of cancer patients was equal to that of the general population. The SMR for accidental death declined within the first five years of cancer diagnosis and then elevated after five years (Supplementary Table.4 ). The highest relative risks of suicide and accidental death persisted over time in patients with type II ovarian cancer.

\section{Discussion}

\section{Main findings}

Using data from the SEER database, we first found that a relative risk of 1.9 for suicide and 1.5 for accidental death among women with ovarian cancers compared with the general US women population. Of note, we found that patients with Type II epithelial ovarian cancer faced an increased risk of both suicide and accidental deaths in comparison with the general population, and the patients with Type I epithelial ovarian cancer also had a greater risk of accidental deaths. Given that suicide is potentially preventable, this analysis is important as it identifies the high-risk groups of death from suicide and accidental injury among ovarian cancer patients, providing a preliminary heuristic framework for clinicians to develop suicide riskidentification and prevention strategies to reduce the risk of suicide among women with ovarian cancer.

\section{Strengths and Limitations}

Despite many important findings of an association between ovarian cancer and risk of suicide and accidental death, there are some limitations in our study. First, the SEER database does not contain detailed infor- 
mation regarding chemoradiotherapy such as cycles and dosage, which renders it difficult to evaluate the impact of chemoradiotherapy on the risk of suicide and accidental death. Second, data on psychiatric status, quality of life, and social support are also unavailable in the SEER database. Third, data on a history of psychiatric disorders, complications of cancer and treatment, the quality of life, and social support are also unavailable in the SEER database, therefore, we cannot assess the direct association between these factors and the risk of suicide and accidental death among patients with ovarian cancer. Nevertheless, this study is the first large population-based study on the risk of suicide and accidental death in patients with ovarian cancer. Hence, the results of the study are reliable and applicable to the rest of the population.

\section{Interpretation}

Some of our research results are very meaningful. Our study showed that patients with Type II epithelial ovarian cancer faced a greater risk of suicidal and accidental mortality which may be attributed to the advanced cancer stage and poor prognosis compared with type I epithelial, germ cell, and sex cord-stromal ovarian cancer ${ }^{16}{ }^{17}$. A Danish study containing 2660 cases reported that $78.1 \%$ of patients with type II epithelial ovarian cancer diagnosed in late cancer stages, with a median follow-up time of 24 months; as a comparison, only $32.1 \%$ of patients with type I epithelial ovarian cancer diagnosed in late cancer stages, with a median follow-up time of 36 months ${ }^{18}$. Using the SEER database, Edward et al. found that the disease-specific survival of type II is being $64-34 \%$ of Type I over the 200 -month range ${ }^{16}$. In the context of the poor prognosis, patients with type II epithelial sarcoma would suffer more psychological concerns, including anxiety and depression. They could feel isolated and hopeless due to the lack of survivor groups. All these may lead to intense suicidal ideation among women with type II epithelial ovarian cancer.

We also found that the risk of suicide was the highest within the first year after initial diagnosis among patients with ovarian cancer. This is a novel finding of the present study, and our results stand corroborated by other cancer studies which showed an elevated risk of suicide within the first year of diagnosis compared with cancer-free controls. Haider et.al reported the highest suicide rates within the first year following diagnosis among women with gynaecologic cancer, with an SMR of $2.8^{8}$. Further, prior research has shown that significantly higher risks of suicide were observed among patients diagnosed with breast cancer ${ }^{19}$, brain cancer $^{20}$, male genital-system ${ }^{21}$, and skin malignant melanoma ${ }^{22}$ within the first year of diagnosis. We thought it reasonable as the change from being a healthy individual to being a cancer patient who had a poor prognosis created a new perception of identity. During this process of shift, patients with ovarian cancer often suffer from severe psychological distress and problems in social life ${ }^{23}$. This novel finding underlines the concept that healthcare providers should better understand the social psychology and identity changes of newly diagnosed patients with ovarian cancer to better guide ovarian cancer survivors during follow-up. Many methods could be utilized to evaluate the level of mental health and suicidal ideation among cancer patients, such as item 9 of the Patient Health Questionnaire depression module, Centre for Epidemiologic Studies Depression Scale (CES-D), the Impact of Event Scale (IES), and the Profile of Mood States shortform (POMS-SF) ${ }^{24}$.

Within the ovarian cancer cohort, we observed that those who underwent pelvic exenteration were at the highest risk of suicide. Pelvic exenteration is a radical surgical procedure that removes the visceral pelvic organs including the uterus, tubes, ovaries, parametrium, vagina, urinary bladder, urethra, and rectum, with or without the perineum in an en-bloc fashion ${ }^{2526} 27$. It is considered the last curative opportunity for malignant gynaecological tumors ${ }^{28}$. However, it is related to various complications, high costs of substantial treatment, and high mortality. A survey conducted in New Zealand reported 106 complications, such as intra-abdominal collection (43.7\%) and wound infection (14.1\%), out of a total of 646 consecutive patients who required extended surgery for local advanced pelvic malignancies ${ }^{29}$. Moreover, severe gastrointestinal and urinary tract symptoms, as well as decreased sexual functions, have also been observed among patients who underwent pelvic exenteration ${ }^{30}$. These adverse effects and accompanying symptoms had an obvious negative impact on global health status, body image, self-identity, social functioning, emotional response, and quality of life among these survivors ${ }^{31}$. Therefore, these survivors who underwent pelvic exenteration have an increased risk of suicide and need close monitoring, physicians should be more mindful of these 
patients to reduce suicidal death.

Ovarian cancer occurs more in patients older than 50 years, similarly, and we found that patients diagnosed over 50 were at higher risk of suicide, especially between the ages of 50-59. Our results stand corroborated by Stephanie's study which found that female patients with cancer whose age at diagnosis between 55 and 59 had the highest suicide risk among all female cancer survivors ${ }^{5}$. Furthermore, it was well recognized that older age was associated with a higher risk of suicide among patients with prostate cancer, lung cancer ${ }^{32}$, colorectal cancer, and bladder cancer ${ }^{33}$. The working hypotheses for our finding may be that ovarian cancer patients with older age may suffer more severe emotional and psychological distress. Payne et al. reported that some symptoms, such as the anxiety and depression of recurrent disease and death, as well as sleep disorders, may persist longer in elder patients ${ }^{34}$. An Australian survey using the Insomnia Severity Index (ISI) to assess the degree of insomnia also confirmed that ovarian cancer patients aged 50-59 present had clinically higher levels of insomnia ${ }^{35}$, which has been proven to be correlated with suicidal thoughts and attempts ${ }^{36}$. Moreover, older patients could get less information about cancer and psychological support than younger patients from the internet ${ }^{3738}$, therefore, they lack the confidence to defeat cancer ${ }^{39}$, leading to relatively higher suicidal ideation ${ }^{40}$. Given the great difference in suicide risk between young and old survivors with ovarian cancer, it is necessary to regard old patients as a distinctive group that warrants special attention when considering the suicide risk of cancer patients.

\section{Conclusion}

The study demonstrated that patients with ovarian cancer, especially type II ovarian cancer, had an increased relative risk of suicide and accidental death to that of the cancer-free population. Further, it is worth noting that pelvic exenteration for ovarian cancer would increase the risk of suicide, but not increase the risk of accidental death. These meaningful findings suggest a need in clinical work to detect suicidal ideation as early as possible especially for high-risk subgroups. Appropriate psychosocial interventions and treatment are essential, such as psychosocial and emotional support through interpersonal interactions during survivorship care, which potentially reduce the risk of suicide in cancer survivors. Future studies should explore the direct relationship between psychosocial factors and the risk of suicide and accidental death among ovarian cancer patients, which may discover new interventions that can improve the survival of patients diagnosed with ovarian cancer.

\section{Disclosure of interests}

No conflicts of interest.

\section{Contribution to authorship}

SW, MW, YC and KY conceived the idea and the study design. QZ, WT, DC and LX performed statistical analysis. YC, KY, QZ and WT wrote the manuscript with revisions and corrections provided by SW, MW, JZ, SZ, JD, YJ. All authors contributed to the interpretation of the findings, critically reviewed the manuscript, and approved the final manuscript version.

\section{Details of ethics approval}

This study was conducted according to the institutional and ethical rules concerning research, and was exempted from patient informed consent.

\section{Funding}

This work was supported by grants from the National Natural Science Foundation of China (no. 81873824 and 82001514).

\section{Acknowledgment:}

The authors acknowledge the efforts of the National Cancer Institute and the Surveillance, Epidemiology, and End Results (SEER) Program tumour registries in the creation of the SEER database. 


\section{Reference:}

1. Centers for Disease Control and Prevention Preventing suicide. National Center for Injury Prevention and Control DoVPRfhwcgvs. [cited; Available from: Retrieved from https://www.cdc.gov/violenceprevention/suicide/fastfact.html

2. Pritchard C, Hean S. Suicide and undetermined deaths among youths and young adults in Latin America: comparison with the 10 major developed countries-a source of hidden suicides? Crisis. 2008;29(3):145-53.

3. Rockett IR, Hobbs G, De Leo D, Stack S, Frost JL, Ducatman AM, et al. Suicide and unintentional poisoning mortality trends in the United States, 1987-2006: two unrelated phenomena? BMC Public Health. 2010 Nov 17;10:705.

4. Bergen H, Hawton K, Kapur N, Cooper J, Steeg S, Ness J, et al. Shared characteristics of suicides and other unnatural deaths following non-fatal self-harm? A multicentre study of risk factors. Psychol Med. 2012 Apr;42(4):727-41.

5. Misono S, Weiss NS, Fann JR, Redman M, Yueh B. Incidence of suicide in persons with cancer. J Clin Oncol. 2008 Oct 10;26(29):4731-8.

6. Yang K, Zheng Y, Peng J, Chen J, Feng H, Yu K, et al. Incidence of Death From Unintentional Injury Among Patients With Cancer in the United States. JAMA Netw Open. 2020 Feb 5;3(2):e1921647.

7. Ward KK, Roncancio AM, Plaxe SC. Women with gynecologic malignancies have a greater incidence of suicide than women with other cancer types. Suicide Life Threat Behav. 2013 Feb;43(1):109-15.

8. Mahdi H, Swensen RE, Munkarah AR, Chiang S, Luhrs K, Lockhart D, et al. Suicide in women with gynecologic cancer. Gynecol Oncol. 2011 Aug;122(2):344-9.

9. Doubeni CA, Doubeni AR, Myers AE. Diagnosis and Management of Ovarian Cancer. Am Fam Physician. 2016 Jun 1;93(11):937-44.

10. Nakao S, Minaguchi T, Itagaki H, Hosokawa Y, Shikama A, Tasaka N, et al. Pretreatment thrombocytosis as an independent predictive factor for chemoresistance and poor survival in epithelial ovarian cancer. J Ovarian Res. 2020 May 6;13(1):55.

11. Dalela D, Krishna N, Okwara J, Preston MA, Abdollah F, Choueiri TK, et al. Suicide and accidental deaths among patients with non-metastatic prostate cancer. BJU Int. 2016 Aug;118(2):286-97.

12. National Cancer Institute. Surveillance E, and End Results Program.SEER*Stat software [computer program]. Version 8.3.6.https://www.seer.cancer.gov/seerstat. Published August 8, 2019. Accessed April 1, 2020. [cited; Available from:

13. Peres LC, Cushing-Haugen KL, Kobel M, Harris HR, Berchuck A, Rossing MA, et al. Invasive Epithelial Ovarian Cancer Survival by Histotype and Disease Stage. J Natl Cancer Inst. 2019 Jan 1;111(1):60-8.

14. Matz M, Coleman MP, Carreira H, Salmeron D, Chirlaque MD, Allemani C, et al. Worldwide comparison of ovarian cancer survival: Histological group and stage at diagnosis (CONCORD-2). Gynecol Oncol. 2017 Feb;144(2):396-404.

15. Koepsell TD WN. Epidemiologic Methods: Studying the Occurrence of Illness. New York: NY: Oxford University Press; 2003.

16. Pavlik EJ, Smith C, Dennis TS, Harvey E, Huang B, Chen Q, et al. Disease-Specific Survival of Type I and Type II Epithelial Ovarian Cancers-Stage Challenges Categorical Assignments of Indolence \& Aggressiveness. Diagnostics (Basel). 2020 Jan 21;10(2).

17. Matz M, Coleman MP, Sant M, Chirlaque MD, Visser O, Gore M, et al. The histology of ovarian cancer: worldwide distribution and implications for international survival comparisons (CONCORD-2). Gynecol Oncol. 2017 Feb;144(2):405-13. 
18. Prahm KP, Karlsen MA, Hogdall E, Scheller NM, Lundvall L, Nedergaard L, et al. The prognostic value of dividing epithelial ovarian cancer into type I and type II tumors based on pathologic characteristics. Gynecol Oncol. 2015 Feb;136(2):205-11.

19. Gaitanidis A, Alevizakos M, Pitiakoudis M, Wiggins D. Trends in incidence and associated risk factors of suicide mortality among breast cancer patients. Psychooncology. 2018 May;27(5):1450-6.

20. Saad AM, Elmatboly AM, Gad MM, Al-Husseini MJ, Jazieh KA, Alzuabi MA, et al. Association of Brain Cancer With Risk of Suicide. JAMA Netw Open. 2020 May 1;3(5):e203862.

21. Yang J, He G, Chen S, Pan Z, Zhang J, Li Y, et al. Incidence and risk factors for suicide death in male patients with genital-system cancer in the United States. Eur J Surg Oncol. 2019 Oct;45(10):1969-76.

22. Yang J, Chen S, Li Y, Wang B, Xin X, Xue X, et al. Incidence rate and risk factors for suicide death in patients with skin malignant melanoma: a Surveillance, Epidemiology, and End Results analysis. Melanoma Res. 2020 Aug;30(4):402-9.

23. Webber K, Carolus E, Mileshkin L, Sommeijer D, McAlpine J, Bladgen S, et al. OVQUEST - Life after the diagnosis and treatment of ovarian cancer - An international survey of symptoms and concerns in ovarian cancer survivors. Gynecol Oncol. 2019 Oct;155(1):126-34.

24. Davis LZ, Cuneo M, Thaker PH, Goodheart MJ, Bender D, Lutgendorf SK. Changes in spiritual wellbeing and psychological outcomes in ovarian cancer survivors. Psychooncology. 2018 Feb;27(2):477-83.

25. de Gregorio N, de Gregorio A, Ebner F, Friedl TWP, Huober J, Hefty R, et al. Pelvic exenteration as ultimate ratio for gynecologic cancers: single-center analyses of 37 cases. Arch Gynecol Obstet. 2019 Jul;300(1):161-8.

26. Matsuo K, Mandelbaum RS, Adams CL, Roman LD, Wright JD. Performance and outcome of pelvic exenteration for gynecologic malignancies: A population-based study. Gynecol Oncol. 2019 May;153(2):36875.

27. Chatterjee S, Chen L, Jones N, Tergas AI, Burke WM, Hou JY, et al. National trends in total pelvic exenteration for gynecologic malignancies. Am J Obstet Gynecol. 2016 Sep;215(3):395-6.

28. Romeo A, Gonzalez MI, Jaunarena J, Zubieta ME, Favre G, Tejerizo JC. Pelvic exenteration for gynecologic malignancies: Postoperative complications and oncologic outcomes. Actas Urol Esp. 2018 Mar;42(2):121-5.

29. Peacock O, Waters PS, Kong JC, Warrier SK, Wakeman C, Eglinton T, et al. Complications After Extended Radical Resections for Locally Advanced and Recurrent Pelvic Malignancies: A 25-Year Experience. Ann Surg Oncol. 2020 Feb;27(2):409-14.

30. Korfage IJ, Essink-Bot M-L, Mols F, van de Poll-Franse L, Kruitwagen R, van Ballegooijen M. Healthrelated quality of life in cervical cancer survivors: a population-based survey. Int J Radiat Oncol Biol Phys. 2009;73(5):1501-9.

31. Dessole M, Petrillo M, Lucidi A, Naldini A, Rossi M, De Iaco P, et al. Quality of Life in Women After Pelvic Exenteration for Gynecological Malignancies: A Multicentric Study. Int J Gynecol Cancer. 2018 Feb;28(2):267-73.

32. Urban D, Rao A, Bressel M, Neiger D, Solomon B, Mileshkin L. Suicide in lung cancer: who is at risk? Chest. 2013;144(4):1245-52.

33. Zaorsky NG, Zhang Y, Tuanquin L, Bluethmann SM, Park HS, Chinchilli VM. Suicide among cancer patients. Nat Commun. 2019 Jan 14;10(1):207.

34. Payne SA. A study of quality of life in cancer patients receiving palliative chemotherapy. Soc Sci Med. 1992;35(12):1505-9. 
35. Price MA, Zachariae R, Butow PN, deFazio A, Chauhan D, Espie CA, et al. Prevalence and predictors of insomnia in women with invasive ovarian cancer: anxiety a major factor. Eur J Cancer. 2009 Dec;45(18):3262-70.

36. Blank M, Zhang J, Lamers F, Taylor AD, Hickie IB, Merikangas KR. Health correlates of insomnia symptoms and comorbid mental disorders in a nationally representative sample of US adolescents. Sleep. 2015;38(2):197-204.

37. Bass SB, Ruzek SB, Gordon TF, Fleisher L, McKeown-Conn N, Moore D. Relationship of Internet health information use with patient behavior and self-efficacy: experiences of newly diagnosed cancer patients who contact the National Cancer Institute's Cancer Information Service. J Health Commun. 2006;11(2):219-36.

38. Schiffman JD, Csongradi E, Suzuki LK. Internet use among adolescent and young adults (AYA) with cancer. Pediatr Blood Cancer. 2008;51(3):410-5.

39. Penn A, Kuperberg A. Psychosocial Support in Adolescents and Young Adults With Cancer. Cancer J. 2018;24(6):321-7.

40. Zhou H, Xian W, Zhang Y, Yang Y, Fang W, Liu J, et al. Suicide among cancer patients: adolescents and young adult (AYA) versus all-age patients. Ann Transl Med. 2019;7(22):658

Table 1. The risk of suicide and accidental death among patients with ovarian cancer diagnosed between January 1, 1973 and December 31, 2016

\begin{tabular}{|c|c|c|c|}
\hline Variable & No. of patients, n (\%) & Person-years & Suicides $^{a}$ \\
\hline & & & No. of deaths, \\
\hline \multicolumn{4}{|r|}{ 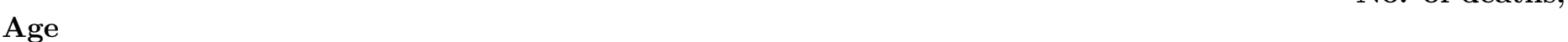 } \\
\hline $0-50$ years old $50+$ years old & $31258(21) 117946(79)$ & 291028.79477811 .38 & $27(25) 80(75)$ \\
\hline \multicolumn{4}{|l|}{ Race } \\
\hline White & $126765(85)$ & 656766.63 & $97(91)$ \\
\hline Black & $11231(8)$ & 47666.21 & $2(2)$ \\
\hline American Indian/AK Native, Asian/Pacific Islander & $10762(7)$ & 61631.83 & $8(7)$ \\
\hline Unknown & $446(0)$ & 2775.50 & $0(0)$ \\
\hline \multicolumn{4}{|l|}{ Year } \\
\hline 1973-1983 & $16667(11)$ & 142227.92 & $23(21)$ \\
\hline 1984-1994 & $21941(15)$ & 166493.13 & $25(23)$ \\
\hline $1995-2005$ & $47111(32)$ & 274347.13 & $30(28)$ \\
\hline $2006-2016$ & $63485(43)$ & 185772.00 & $29(27)$ \\
\hline \multicolumn{4}{|l|}{ Education e } \\
\hline High & $46980(31)$ & 264741.88 & $42(39)$ \\
\hline Medium & $52495(35)$ & 269128.08 & $35(33)$ \\
\hline Low & $49729(33)$ & 234970.21 & $30(28)$ \\
\hline \multicolumn{4}{|l|}{ Income $^{\text {e }}$} \\
\hline High & $49603(33)$ & 276519.21 & $43(40)$ \\
\hline Medium & $45261(30)$ & 238441.83 & $30(28)$ \\
\hline Low & $54340(36)$ & 253879.13 & $34(32)$ \\
\hline \multicolumn{4}{|l|}{ Residence } \\
\hline Metropolitan & $131445(88)$ & 673584.54 & $97(91)$ \\
\hline Non-metropolitan & $16093(11)$ & 79892.33 & $6(6)$ \\
\hline Unknown & $1666(1)$ & 15363.29 & $4(4)$ \\
\hline \multicolumn{4}{|l|}{ Grade $^{f}$} \\
\hline Low & $29892(20)$ & 248906.46 & $18(17)$ \\
\hline High & $59048(40)$ & 251698.00 & $45(42)$ \\
\hline Unknown & $60264(40)$ & 268235.71 & $44(41)$ \\
\hline
\end{tabular}




\begin{tabular}{llll}
\hline Variable & No. of patients, n (\%) & Person-years & Suicides $^{\text {a }}$ \\
\hline Stage & & & \\
Localized & $30442(20)$ & 325990.54 & $32(30)$ \\
Regional & $17588(12)$ & 105362.54 & $16(15)$ \\
Distant & $91669(61)$ & 305955.33 & $49(46)$ \\
Unknown & $9505(6)$ & 31531.75 & $10(9)$ \\
Surgery & & & \\
None & $29246(20)$ & 35362.29 & $15(14)$ \\
Debulking & $36959(25)$ & 140717.75 & $20(19)$ \\
Oophorectomy without hysterectomy & $14606(10)$ & 102602.71 & $7(7)$ \\
Oophorectomy with hysterectomy & $41072(28)$ & 293544.42 & $33(31)$ \\
Pelvic Exenteration & $2370(2)$ & 10111.83 & $3(3)$ \\
Surgery, NOS & $17833(12)$ & 173819.00 & $22(21)$ \\
Unknown & $7118(5)$ & 12682.17 & $7(7)$ \\
Chemotherapy & & & \\
None/Unknown & $58447(39)$ & 339837.00 & $52(49)$ \\
Yes & $90757(61)$ & 429003.17 & $55(51)$ \\
Radiation & & & \\
None/Unknown & $143841(96)$ & 723213.58 & $99(93)$ \\
Yes & $5363(4)$ & 45626.58 & $8(7)$ \\
All & 149204 & 768840.17 & $107(100)$ \\
\hline
\end{tabular}

Abbreviations: SMR, standardized mortality ratio; CI, confidence intervals.

${ }^{a}$ International Classification of Diseases, Eighth Revision codes (ICD-8): 950-959; International Classification of Diseases, Ninth Revision codes (ICD-9): 950-959; and International Statistical Classification of Diseases and Related Health Problems, Tenth Revision (ICD-10) codes U03, X60-X84, and Y87.0 and recode 50220 .

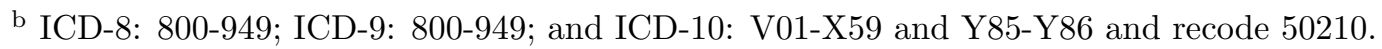

${ }^{c}$ Per 100,000 person-years.

${ }^{\mathrm{d}}$ In brief, SMRs were estimated as the ratios of observed to expected number of deaths. The observed number of deaths represents the total number of deaths from suicide or accidental injury among patients with primary ovary cancer. To obtain the expected number of deaths, we derived the stratum-specific mortality rates of suicide or accidental injury of the general US population and calculated the person-years of relevant strata in the cancer group. The stratum-specific expected number of deaths was estimated as the product of mortality rate in the cancer-free control and the person-years in the cancer group. The total expected number of deaths was a summation of all the expected number of deaths across the strata.

${ }^{\mathrm{e}}$ Income (median family income) and educational level (percentage of residents $>25$ years of age with at least a bachelor's degree) were categorized into quartiles.

f The variable of low grade included "Well differentiated; Grade I" and "Moderately differentiated; Grade II", and the variable of high grade included "Poorly differentiated; Grade III" and "Undifferentiated; anaplastic; Grade IV".

Table 2. The risk of suicide and accidental death among patients with ovarian cancer by histological subgroup.

\begin{tabular}{lllll}
\hline Histological group & No. of patients, n (\%) & Person-years & Suicides & Suicides \\
\hline & & & No. of deaths, n (\%) & Mortality \\
Type I epithelial & $34289(23)$ & 272065.13 & $24(22)$ & 8.82
\end{tabular}




\begin{tabular}{lllll}
\hline Histological group & No. of patients, n (\%) & Person-years & Suicides & Suicides \\
\hline Low-grade serous carcinoma & $980(1)$ & 4423.38 & $1(1)$ & 22.61 \\
Clear cell carcinoma & $6598(4)$ & 45358.08 & $2(2)$ & 4.41 \\
Endometrioid carcinoma & $14699(10)$ & 124382.46 & $10(9)$ & 8.04 \\
Mucinous carcinoma & $10885(7)$ & 91424.29 & $10(9)$ & 10.94 \\
Squamous carcinoma & $489(0)$ & 2336.75 & $0(0)$ & 0.00 \\
Transitional cell or Brenner carcinoma & $638(0)$ & 4140.17 & $1(1)$ & 24.15 \\
Type II epithelial & $102418(69)$ & 411700.75 & $72(67)$ & 17.49 \\
High-grade serous carcinoma & $58242(39)$ & 282216.67 & $43(40)$ & 15.24 \\
Mixed epithelial-stromal carcinoma & $7464(5)$ & 29225.54 & $5(5)$ & 17.11 \\
Undifferentiated or other epithelial & $36712(25)$ & 100258.54 & $24(22)$ & 23.94 \\
Germ cell & $4436(3)$ & 51028.00 & $4(4)$ & 7.84 \\
Sex cord-stromal & $2620(2)$ & 24162.46 & $4(4)$ & 16.55 \\
Others & $5441(4)$ & 9883.83 & $3(3)$ & 30.35 \\
Non-specific & $5075(3)$ & 8386.29 & $2(2)$ & 23.85 \\
Other specific non-epithelial & $366(0)$ & 1497.54 & $1(1)$ & 66.78 \\
\hline
\end{tabular}

Abbreviations: SMR, standardized mortality ratio; CI, confidence intervals.

\section{Figure Legends:}

Figure.1 The SMRs with 95\% CIs for patients with different subtype of ovarian cancer. The lower limit of the CI exceeding 1 means that the patients had a higher risk of suicide (a) or accidental death (b) compared with the general population.

Figure.2 Cumulative probability of suicide (a) and accidental death (b) in women with ovarian cancer who received different types of surgery.

Supplementary Figure.1 Flow diagram of patient selection within the SEER 18 registries Between 1973 and 2016 .

Supplementary Figure.2 Deaths due to suicide and accidental injury among patients with primary malignant ovarian cancer in SEER database by calendar year of death.

Supplementary Figure.3 The number of suicides and accidental death among patients with ovarian cancer by age at diagnosis. 


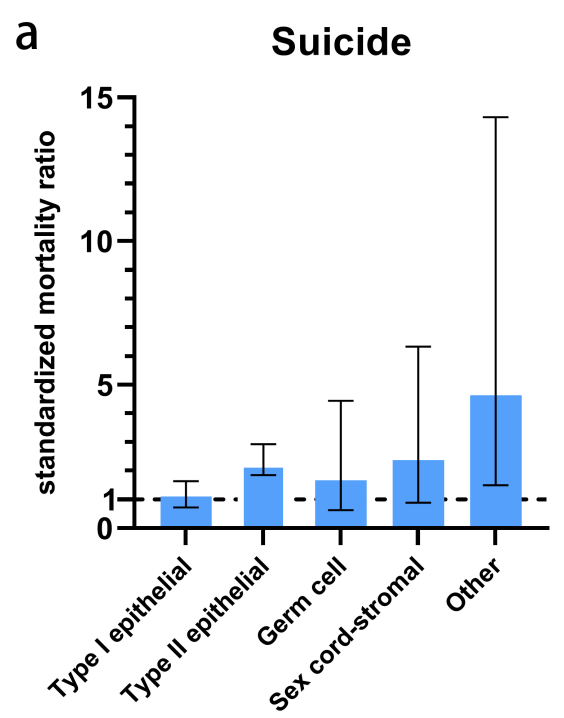

Histological subtype

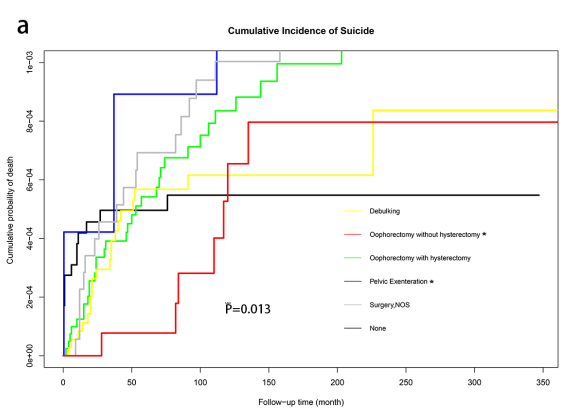

b Accidental Death

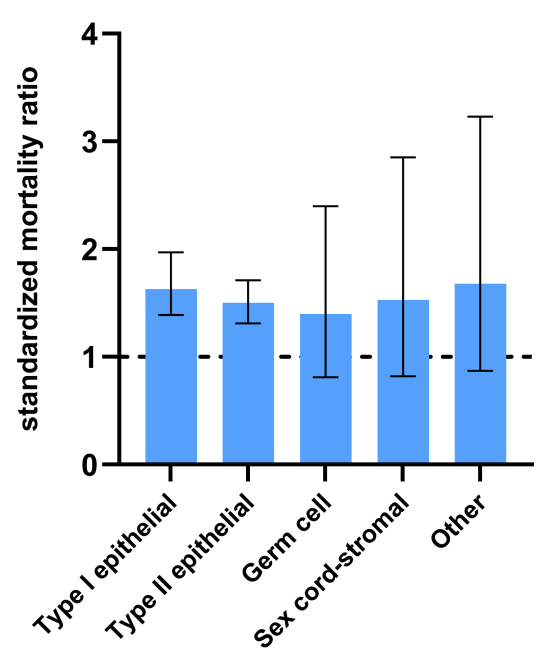

Histological subtype

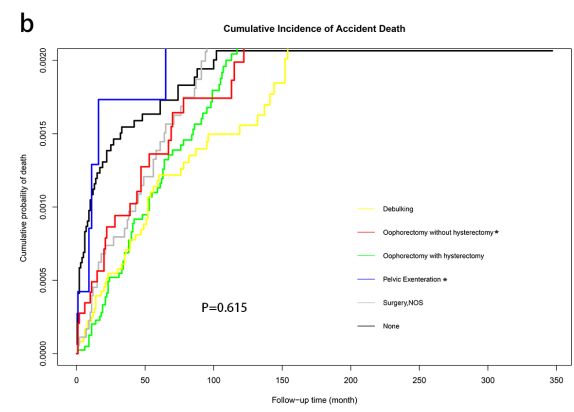

